\title{
A Systematic Review of File Sharing in Mobile Devices Using Peer-To-Peer Systems
}

\author{
Waheed Yasin (Corresponding author) \\ Faculty of Computer Science and Information Technology \\ Universiti Putra Malaysia \\ 43400 UPM, Serdang, Selangor Darul Ehsan, Malaysia
}

Tel: 60-17-294-9240 E-mail: waheedos80@yahoo.com

Hamidah Ibrahim

Faculty of Computer Science and Information Technology

Universiti Putra Malaysia

43400 UPM, Serdang, Selangor Darul Ehsan, Malaysia

Tel: 60-3-8946-8946-6510 E-mail: hamidah@fsktm.upm.edu.my

Nor Asila Wati Abdul Hamid

Faculty of Computer Science and Information Technology

Universiti Putra Malaysia

43400 UPM, Serdang, Selangor Darul Ehsan, Malaysia

Tel: 60-3-8946-8947-1749 E-mail: asila@fsktm.upm.edu.my

\section{Nur Izura Udzir}

Faculty of Computer Science and Information Technology

Universiti Putra Malaysia

43400 UPM, Serdang, Selangor Darul Ehsan, Malaysia

Tel: 60-3-8946-8946-6521 E-mail: izura@fsktm.upm.edu.my

\begin{abstract}
Deployment of file sharing systems on wireless networks introduces several challenges specially when mobility comes into view. Thus, many researches have been performed in this field. The aim of this paper is to perform a systematic review on the existing solutions for file sharing in mobile devices based on Peer-to-Peer or shortly P2P approach. Five approaches are presented that are based on unstructured P2P systems; structured P2P system; mobile-to-mobile technique; tree-structured based; and incentive. These approaches of file sharing can work on one of the two wireless network configurations which are Mobile Ad Hoc Network (MANET) and infrastructure based network. Furthermore, we discussed the advantages and limitations of these approaches. Finally, this paper concludes with some insights for research directions in this area.
\end{abstract}

Keywords: Peer-to-Peer, Mobile Ad Hoc, Structured P2P, Unstructured P2P, Distributed Hash Table

\section{Introduction}

File sharing is the process of making files (e.g. documents, audio, video, etc) available to others. This service is easily available in wired networks; however due to the fast growth of wireless networks, it is not possible to provide only a fixed access to the users. On the other hand, Personal Digital Assistants (PDAs) became so developed and have almost the same abilities of desktop computers. A key point has to be mentioned is that users are moving to a more and more mobile-connected world (Molina, Pileggi, Esteve, \& Palau, 2009). The challenge is how to provide file sharing on mobile devices.

There are two different ways for configuring a mobile network which are infrastructure based and Ad hoc (Chan, $\mathrm{Li}, \&$ Chen, 2009). The infrastructure based needs a preexisting infrastructure such as the availability of Access 
Points (APs); however, this is not applicable in such network where the users connect and disconnect dynamically. Such system called Mobile Ad hoc Network (MANET), where there is no need for preexisting infrastructures.

Although, mobile devices became so developed, they have some limitations such as:

- Limited processing power

- Limited memory capacity

- Operator constraints

- Some of them may have different capabilities

There are several connectivity options available in wireless environment such as General Packet Radio Service (GPRS) (Ghribi \& Logrippo, 2000), Bluetooth (http://www.bluetooth.com), Wi-Fi (http://www.wifinotes. com/IEEE-802.11.html), and Universal Mobile Telecommunications System (UMTS) (http://www.umtsinfo. co.uk/umtsstandards.html). The challenge is how to provide file sharing in mobile environment. Thus, many approaches have been adopted to provide file sharing for mobile devices, such as Peer-to-Peer (P2P) approach, which is the most popular approach.

The aim of this paper is to review the technologies of file sharing in two areas of networks, MANET and mobile devices based on P2P approach.

This paper is organized as follows: In section 2, Peer-to-Peer approach is briefly presented. The existing solutions for file sharing in mobile environment are presented in section 3. In section 4, these solutions are compared according to our discussion. In section 5, the conclusion of this paper is summarized; also insights of research directions in this area are given.

\section{P2P Approach}

P2P defines a way to communicate between two computers in which the same protocol is used, and each computer has equivalent capabilities and responsibilities. Several protocols have been proposed for P2P approach such as Pastry (Gkantsidis \& Rodriguez, 2005) and CAN (Katti et al., 2008); however, these protocols may not be reliable in wireless environment, due to some reasons such as high failure rates, excessive overheads, and mobility (X. Li \& Ji, 2009).

Figure 1 illustrates a P2P overlay on a MANET. The black circles refer to the nodes which are joining the overlay network, while the others are not joining the overlay. The dashed lines show logical links in the overlay network.

\section{P2P on Mobile Devices}

P2P networks are decentralized and dynamic, which make it suitable for MANET. Also, there are some advantages of P2P architecture that make it a suitable network technology for mobile systems such as reliability, easy one to one information exchanging, and distribution of resources (Abiona et al., 2009).

A user may ask why a P2P system is desirable, while he can easily exchange files with others through Bluetooth connectivity. The answer is that Bluetooth connectivity is established only between two users who know each other and decide to exchange files between their devices after comparing their contents.

P2P systems enable mobile peers to establish communications over wireless links based on proximity (Palazzi, Bujari, \& Cervi, 2009). For more clarification, assume that a user $A$ has a file $x$ and wants to get a file $y . \quad A$ usually takes a train to go to his office. During that he passes by a lot of other device users. Imagine that $A$ will pass by a user $B$, who has the file $y$ in his device or may sit next to a user $C$, who wants to get the file $x$. Because of their closeness, it would be easy for a suitable file sharing application to use the Bluetooth connectivity on mobile devices for the users $A, B$ and $C$ to exchange $x$ and $y$ files. Design issues and implementations details of such P2P file sharing applications have been proposed in (Palazzi et al., 2009), which is called Mobile-to-Mobile Share (M2MShare). This application is based on Java J2ME. M2MShare adopted Bluetooth connectivity rather than Wi-Fi technology due to power consumption reasons, where it has been found that Bluetooth consumes less than $200 \mathrm{~mW}$; while Wi-Fi consumes more than $650 \mathrm{~mW}$, when employing two communication chips which are CSR BlueCore2 and Conexant CX53111. Figure 2 illustrates a power consumption comparison between Bluetooth and Wi-Fi (Palazzi et al., 2009). M2MShare has some limitations such as it does not provide the ability of interrupt and resume downloads. Also, M2MShare shares a file as one unit. That means if the file is available on many mobile devices, it can be downloaded only from one mobile device.

In this section, we review five different $\mathrm{P} 2 \mathrm{P}$ approaches which have been adopted in mobile environment, that are unstructured-based P2P approach, structured-based P2P approach, tree-structure based P2P approach using 
distributed hash table, P2P systems based on direct mobile-to-mobile technique, and finally P2P systems based on incentive approach.

\subsection{P2P Systems Based on Unstructured Approach}

Peers in unstructured P2P systems are unaware of resources neighboring peers; instead of that they determine search requests using flooding techniques (Jin \& Jiang, 2007).

Gnutella (S. -. R. Li, Yeung, \& Ning Cai, 2003) is the most popular protocol of unstructured P2P, which is more flexible than the structured P2P protocol when it is used in mobile environment (Ting Li, Hong Ji, Jingqing Mei, Yi Li, \& Chao Hu, 2009); however message redundancy is one of its drawbacks.

The proposed mechanism in (Ting Li et al., 2009) has applied Gnutella as the basic P2P protocol to construct a file sharing system. In this paper we call this proposed mechanism as TingLi2009. The control overhead which is imposed by Gnutella in mobile environment has been also analyzed in (Ting Li et al., 2009). Establishing connection operations in Gnutella consume bandwidth. Table I shows the parameters that are used to estimate the bandwidth consumption for establishing a connection.

The total number of messages $T_{\text {total }}$ originated from one discovery Ping, including the respondent Pongs, can be calculated using equation (1) where $h_{T T L}$ refers to the Time to Live (TTL) of discovery Ping message, $c$ indicates the connection degree of peers, which is also the maximum value of the connections at each peer.

$$
\mathrm{T}_{\text {total }}=2 \sum_{\mathrm{i}=0}^{\mathrm{b}=\mathrm{i}-1} \mathrm{c}(\mathrm{c}-1)^{\mathrm{i}}
$$

Figure 3 illustrates a Gnutella file discovery process, and presents an example of zigzag routing problem (Corberán, Mota, \& Sanchis, 2006), where node 1 requires the file "bestmusic.mp4". Thus, it first sends a query message to its logical neighbor node 3 . After receiving the query message, node 3 looks into the local storage according to the information contained in the query message. Because of the requested file does not exist in node 3 , the query message is forwarded to node 4 . At node 4, the requested file also does not exist; thus, the query message is forwarded to node 7. The query message will be forwarded since the value of its TTL field is greater than 0 . Eventually, the file "bestmusic.mp4" is found at node 7. Then node 7 replies a Query-Hit message back to node 1. In the end of file discovery procedure, node 1 could perform download procedure. The logical connection for the query message is "1-3-4-7"; however, a P2P connection is created on several physical hops. Thus, to transmit a message from one peer to another, it has to pass through intermediate nodes in the network.

As illustrated in Figure 3, the physical path followed by the query message from node 1 to node 7 is "1-2-3-5-4-5-6-7". The link between node 4 and node 5 is always passed twice, and it is the zigzag routing problem.

As we mentioned earlier maintaining P2P connection generates traffic which may consume up to $34 \%$ of bandwidth (Ting Li et al., 2009), which is considered as the bottleneck for deploying Gnutella file sharing system over MANETs especially when there is a heavy traffic. The cross-layer protocol proposed in TingLi2009 mechanism is based on the reactive routing protocols in MANETs, and it has been compared to off-the-shelf protocol which represents the system without any enhancement.

Figure 4 illustrates the traffic generated by the P2P application at the MAC layer. It shows that TingLi2009 mechanism induces less control overhead than that of off-the-shelf approach for file discovery.

\subsection{P2P Systems Based on Structured Approach}

Peers in structured P2P systems maintain information about resources offered by neighbors. Thus fewer messages are needed for answering queries. Moreover, structured P2P systems provide both low latency and load balancing. These systems are based on Distributed Hash Table (DHT) in which hash functions are used to map peers and shared content references. Structured P2P networks cause high signaling overhead.

Chord (Ho et al., 2006) is a protocol which belongs to the class of structured P2P systems. DHT is a key point in Chord system which, uses one dimensional $\mathrm{m}$-bit hash function and organizes peers using ring topology. Figure 5 illustrates a Chord Identifier in a P2P network where a part of ID space between a peer ID and its predecessor ID is the responsibility of each peer in the network. In order to answer queries, all peers have to identify at least their successor on the ring. Then messages can be routed with a simple routing system that does not scale. This drawback might result in using additional routing information which is called fingers that are stored at every single peer. Using equation (2), the fingers for peer $n$ can be calculated (Kellerer, Kunzmann, Schollmeier, \& Zöls, 2006).

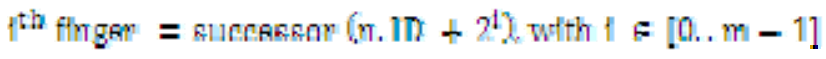


A structured P2P networks for telecommunications and mobile environments based on Chord protocol using DHT have been presented in (Kellerer et al., 2006). In this paper we call it Wolfgang2006 mechanism.

Structured P2P networks have some limitations such as causing high signaling overhead in highly dynamic networks with high churn rates, even if the participating peers do not initiate search requests. Wolfgang2006 mechanism proposed a Token Chord, which has some advantages such as stabilization especially for high churn rates, while, standard Chord is not able to update its successor lists rapidly. Figure 6 illustrates the performance of Token Chord compared to the Standard Chord.

From Figure 6, it can be observed that the number of errors in the successor lists is noticeably smaller when token-based stabilization mechanism is used.

\subsection{P2P Systems Based on Direct Mobile-to Mobile Technique}

In order to decrease structured P2P overhead, enhancements of structured P2P approaches to increase stability and availability in structured P2P network have been presented in (Popova, Herpel, Gerstacker, \& Koch, 2008). These enhancements are based on exchanging data cooperatively between users in direct mobile-to-mobile (M2M2008) technique over available UMTS uplink channels, and integrating a P2P technique in UMTS networks. In (Popova et al., 2008), in order to enable the cooperative P2P technique in wireless networks, the idea of mesh architecture has been extended to Frequency Division Duplex (FDD). In this paper, this technique is called as M2M2008. The concept of M2M2008 technique is illustrated in Figure 7 considering an example that mobile terminals (MTs) participate freely in file sharing.

Each new user who is interested in downloading a certain file (e.g. "bestmusic.mp4") establishes a connection to the Base Station (BS) and Radio Network Controller (RNC) in order to participate in M2M2008 file transfer. Also, in order to get a random packet from "bestmusic.mp4", as well as information about neighboring M2M2008 peers, which are interested in "bestmusic.mp4" where either "Hello" packets, that may consumes bandwidth, or GPS (Global Positioning System) techniques, which provides information about M2M2008 peers whom exist in a certain range of the system, is used. Figure 8 illustrates the basic steps to establish a cooperative M2M2008 connection.

A key point has to be mentioned about M2M2008 technique is that M2M2008 peers must be organized into groups. The group size is limited and each MT can be a member of only one group at a time, and members are not constrained to be from the same cell.

Figure 9 illustrates a comparison of M2M2008 file sharing with conventional UMTS data transmission in high load (Popova et al., 2008). It can be observed that there is a significant reduction of the expected file download time, which is $142.3 \mathrm{sec}$.

\subsection{P2P Systems Based on DHT and Tree-Structure Approach}

A file sharing system has been proposed for MANET in (Sözer, Tekkalmaz, \& Korpeoglu, 2009) based on P2P approach, which is called in this paper as HMI2009 system. HMI2009 system uses a DHT and a tree-structure based on the topology of the network in order to store and maintain peers' locations and routing information.

The underlying network layers are responsible to carry out the following basic functions, which are expected to be performed by HMI2009 system.

- Node detection

- Nodes communication within the same range

- Failure notification

In addition to these functions, HMI2009 system utilizes a completely DHT which its keys include shared files' names where these values are unique. For example, node Media Access Control (MAC) address and file's path on that node might be considered as a way to grant values uniqueness. Also, DHT keys include important routing information.

The basic mechanism of HMI2009 can be summarized as follows: (key, value) pairs are stored in a space with one dimension using uniform hash function to map each key on the "hashline" to a point $X$. In order to carry out this mapping, any hash might be used provided that this function is able to map the file name to a number within the range 0 and 1 . However, the uniformity of the function might increase the fairness of information distribution between nodes. All nodes in the MANET are responsible to store a partition of the hashline. That means the DHT records which match points that are belonged to this hashline partition. 
In this paper, the node which is responsible for a partition of the hashline including the point $X$ is called $X$-Node. Also, we called the node which stores a file which its name is e.g. "bestmusic.mp4" as bestmusic.mp4-Node. For more clarification, $X$-Node keeps information such as index and location, while the file "bestmusic.mp4" is stored in bestmusic.mp4-Node.

In HMI2009, the following steps are required to access a file:

- Hashing the name of the required file ("bestmusic.mp4" in our case) in order to identify point $X$ on the hashline.

- Accessing Point $X$.

- Determining the location of the file "bestmusic.mp4", and the route to this location.

- Accessing bestmusic.mp4-Node.

- Downloading the file.

The most important part of HMI2009 system is identifying the routes between nodes. HMI2009 system has been proposed based on logical tree-structure approach in order to handle problems such as determining routes between nodes in MANET. For example, in order to access $X$-Node, this logical tree-structure approach can play a main role in performing that. Also, in order to determine the route to bestmusic.mp4-Node for file downloading, the information which is obtained from $X$-Node will assist in performing that.

A key point has to be mentioned about HMI2009 system is that it assumes that loops are not allowed at application layer. Another assumption in HMI2009 system is that new nodes are not allowed to share the same file through more than one neighboring node. This assumption could be satisfied by applying network ID, and each node having this network ID has only one parent.

Figure 10 illustrates an example of using HMI2009 in MANET with six nodes. In this example, we assume that node 3 searches for the file "bestmusic.mp4". This file is stored in node 5. Thus, node 5 is called bestmusic.mp4-Node. This file is mapped to a point $X$ on partition of the hashline which is responsible by node 4 . Thus, node 4 is the $X$-Node which determines the route from itself to the bestmusic.mp4-Node, node 5. First, in order to download the file "bestmusic.mp4", node 3 has to reach node 4 . Then, it can determine the route to node 5 where the file is located.

As it has been mentioned earlier, HMI2009 system applies logical tree-structure approach in its network. As a result of that, each parent has to know its children, in addition to the hashline partitions which it is responsible for. For example, node 3 does not have children, and it knows that $X$-Node does not belong to the same hashline partition. Thus, it forwards query message to its parent, node 1 . When node 1 gets this query, it forwards the query to node 4 ( $X$-Node) because the responsibility of the corresponding hashline segment was previously delegated to this node. As node 1 receives query message, it forwards this message to node 4 ( $X$-Node), because this node is responsible for the hashline partition. Eventually, node 4 ( $X$-Node) gives the route information to node 5 (bestmusic.mp4-Node).

\subsection{P2P Systems Based on Incentive Approach}

An incentive mechanism is based on encouraging sharing and forwarding of data between intermediate nodes. A proposed mechanism called Incite has been presented in (Mawji \& Hassanein, 2009). In this paper we call this mechanism as Incite2009.

Incite2009 implies cooperation between nodes based on their energy, and the performance (reputation) of the requesting node. Each node has a performance index which is based on how many times it has cooperated with others in both of sharing files and forwarding data. If the node has a low level of reputation, it has an incentive to cooperate in order to increase its reputation level.

Assume that Client-Node refers to the node which wants to download a file, and Sever-Node refers to the node that is having this file. Client-Node sends a Request-Message to Server-Node including its reputation index. If the Server-Node decides to cooperate, it replies with an Agree-Message; else, it replies with a Disagree-Message. When the Server-Node decides to cooperate, it has to provide the Client-Node with the route information. This procedure is performed by sending Forward-Message to any neighbor of Client-Node provided that this neighbor is ready to cooperate. This procedure is repeated until the Client-Node is contacted. At this point, the Client-Node sends Enqueue-Message to the Server-Node indicating that it is ready to download the file. If the path between Client-Node and Server-Node was not discovered, the last neighbor which agreed to cooperate sends a Failed-Message to the Client-Node. Figure 11 presents the algorithm of Incite2009 mechanism. 
To determine reputation information, a calculation is made by nodes depending only on their local information, which results in reducing both the number of messages, and energy consumption. For example, assume that $X$-Node is the sender node, and $Y$-Node is the receiver node. If $\mathrm{X}_{\text {Reputation }}+t \geq \mathrm{Y}_{\text {Reputation, }}$, where $\mathrm{X}_{\text {Reputation }}, \mathrm{Y}_{\text {Reputation }}$ are the reputation indexes of $X$-Node, $Y$-Node respectively, and $t$ is the threshold window of the reputation. $Y$-Node cooperation probability can be calculated using equation (3).

$$
\text { Prob }_{\text {coop }}=E_{Y} \times X_{\text {Reputation }}, 0 \leq E_{Y} \leq 1,0 \leq X_{\text {Reputation }} \leq 1
$$

where $\mathrm{E}_{\mathrm{Y}}$ is the lasting energy of $Y$-Node, and $\mathrm{X}_{\text {Reputation }}$ is the reputation index of $X$-Node. If the reputation is below $t$, the probability of cooperation is determined by equation (4).

$$
\text { Prob }_{\text {cocp }}=\operatorname{Max} \times \frac{-1}{\operatorname{maxas} E_{a x}} \quad, 0 \leq E_{Y} \leq 1,0 \leq \operatorname{Max} \leq 1
$$

where Max is the maximum probability needed for cooperation and $\mathrm{E}_{\mathrm{Y}}$ is the remaining energy of $Y$-Node. The reputation index for any node (e.g. $X$-Node) can be calculated using equation (5).

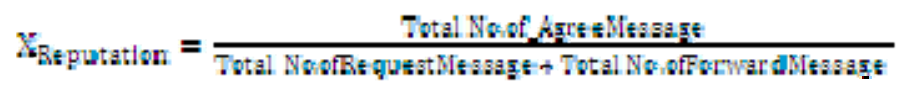

Figure 12 illustrates the performance of Incite2009 mechanism using different values of threshold window $t$ taking the average number of downloads per node; also it is compared to P2P network with different ratios of altruistic and freeloading peers. It can be observed that Incite2009 increases the average number of downloads per node as the threshold window $t$ increases; however selecting the best value of $t$, under which circumstances has not been discussed in (Mawji \& Hassanein, 2009). Also it has been assumed that nodes provide their reputation information honestly without considering security issues.

\section{Comparison of File Sharing Mechanisms in Mobile Environment}

In this section we will make a comparison between all the techniques which have been covered in this paper.

Peers in unstructured P2P systems are unaware of resources neighboring peers; instead of that, they determine search requests using flooding techniques; while, peers in structured P2P systems maintain information about resources offered by neighbors. Thus fewer messages are needed for answering queries, and this will save the bandwidth. Despite of that, this causes overhead and leads to consume the bandwidth in signaling rather than sending the actual data.

Message redundancy is one of Gnutella drawbacks of which has been applied as the basic P2P protocol to construct a file sharing system in TingLi2009. Another drawback is the control overhead which is imposed by Gnutella in mobile environment.

One of the drawbacks of M2M2008 technique is that it is based on social principle, which means a user must allow the use of his currently available uplink capacity by providing the packets of the content which he requested to other M2M2008 users that are interested in it (service level agreement).

Also M2M2008 can only be applied in sharing non-real-time data content, which can be considered as another drawback.

However HMI2009 system performs better than TingLi2009 system. It has some limitations such as it may not work efficiently when frequent disconnections happen. Also, it is based on hash function which one of its drawbacks is the possibility of searching only for exact file names. For P2P systems which are based on incentive approach, it can be observed that Incite2009 increases the average number of downloads per node as the threshold window $t$ increases; however selecting the best value of $t$, under which circumstances has not been discussed in (Mawji \& Hassanein, 2009).

Table II lists a comparison between file sharing techniques which have been reviewed in this paper.

\section{Conclusion}

Deployment of file sharing systems on wireless networks introduces several challenges. Thus, many researches have been performed in this field. In this paper, a systematic review on five approaches for file sharing in mobile devices using P2P systems has been performed. These approaches are based on unstructured P2P systems; structured P2P system; mobile-to-mobile technique; tree-structured based; and incentive. Furthermore, we discussed the advantages and limitations of these solutions. Also, a comparison between those solutions has been performed. As future works, the messaging mechanism used to establish a connection in unstructured P2P can be modified. Also, the DHT function can be adjusted in both structured and tree-structured P2P systems. Thus, it is 
not only searching for exact file names. Another enhancement is how to make the system stable even when frequent disconnections happen. An enhancement can be made for M2M2008 technique based on the type of application that can be shared such as real time applications. For Incite2009 mechanism, determining the best value of window threshold can be considered as an enhancement for Incite2009.

An appropriate integration of file sharing using P2P techniques may reduce the delay experienced by users during accessing web sites. Also, this kind of integration will save the bandwidth from repeated traffic and signaling overhead.

\section{References}

Abiona, O. O., Oluwaranti, A. I., Anjali, T., Onime, C. E., Popoola, E. O., Aderounmu, G. A., et al. (2009). Architectural model for wireless peer-to-peer (WP2P) file sharing for ubiquitous mobile devices. IEEE International Conference on Electro/Information Technology, 35-39.

Chan, E., Li, W., \& Chen, D. (2009). Energy saving strategies for cooperative cache replacement in mobile ad hoc networks. Pervasive and Mobile Computing, 5(1), 77-92.

Corberán, A., Mota, E., \& Sanchis, J. M. (2006). A comparison of two different formulations for arc routing problems on mixed graphs. Computers \& Operations Research, 33(12), 3384-3402.

Ghribi, B., \& Logrippo, L. (2000). Understanding GPRS: The GSM packet radio service. Computer Networks, 34(5), 763-779.

Gkantsidis, C., \& Rodriguez, P. R. (2005). Network coding for large scale content distribution. Proceedings of the 24th Annual Joint Conference of the IEEE Computer and Communications Societies, 4 2235-2245.

Ho, T., Medard, M., Koetter, R., Karger, D. R., Effros, M., Jun Shi, et al. (2006). A random linear network coding approach to multicast. IEEE Transactions on Information Theory, 52(10), 4413-4430.

Jin, S., \& Jiang, H. (2007). Novel approaches to efficient flooding search in peer-to-peer networks. Computer Networks, 51(10), 2818-2832.

Katti, S., Rahul, H., Wenjun Hu, Katabi, D., Medard, M., \& Crowcroft, J. (2008). XORs in the air: Practical wireless network coding. IEEE/ACM Transactions on Networking, , 16(3) 497-510.

Kellerer, W., Kunzmann, G., Schollmeier, R., \& Zöls, S. (2006). Structured peer-to-peer systems for telecommunications and mobile environments. AEU - International Journal of Electronics and Communications, 60(1), 25-29.

Li, S. -. R., Yeung, R. W., \& Ning Cai. (2003). Linear network coding. IEEE Transactions on Information Theory, 49(2), 371-381.

Li, X., \& Ji, H. (2009). Scalable peer-to-peer resource discovering scheme for wireless self-organized networks. The Journal of China Universities of Posts and Telecommunications, 16(2), 47-52, 57.

Mawji, A., \& Hassanein, H. (2009). Incentives for P2P file sharing in mobile ad hoc networks. 6th IEEE Consumer Communications and Networking Conference, 1-5.

Molina, B., Pileggi, S. F., Esteve, M., \& Palau, C. E. (2009). A negotiation framework for content distribution in mobile transient networks. Journal of Network and Computer Applications, 32(5), 1000-1011.

Palazzi, C. E., Bujari, A., \& Cervi, E. (2009). P2P file sharing on mobile phones: Design and implementation of a prototype. 2nd IEEE International Conference on Computer Science and Information Technology, 136-140.

Popova, L., Herpel, T., Gerstacker, W., \& Koch, W. (2008). Cooperative mobile-to-mobile file dissemination in cellular networks within a unified radio interface. Computer Networks, 52(6), 1153-1165.

Sözer, H., Tekkalmaz, M., \& Korpeoglu, I. (2009). A peer-to-peer file search and download protocol for wireless ad-hoc networks. Computer Communications, 32(1), 41-50.

Ting Li, Hong Ji, Jingqing Mei, Yi Li, \& Chao Hu. (2009). Topology mismatch avoidable cross-layer protocol for P2P file discovery in MANETs. Wireless Communications and Networking Conference, 2009. WCNC 2009. IEEE, $1-5$. 
Table 1. Parameters of Gnutella P2P protocol (Ting Li et al., 2009)

\begin{tabular}{|l|l|l|}
\hline Parameter & Value & Unit \\
\hline Probe ping interval & 30 & second \\
\hline Discovery ping interval & 5 & second \\
\hline Probe ping retires & 2 & number \\
\hline Peer connection degree & 4 & number \\
\hline Discovery ping TTL & 7 & number \\
\hline Probe ping TTL & 1 & number \\
\hline Ping & 22 & byte \\
\hline Pong & 35 & byte \\
\hline
\end{tabular}

Table 2. Comparison between file sharing techniques

(format the table using distribute columns evenly)

\begin{tabular}{|c|c|c|c|c|c|}
\hline Mechanism & TingLi2009 & Wolfgang2006 & M2M2008 & HMI2009 & Incite2009 \\
\hline Approach & $\begin{array}{l}\text { Based on } \\
\text { unstructured } \\
\text { approach using } \\
\text { Gnutella } \\
\text { approach }\end{array}$ & $\begin{array}{l}\text { P2P systems } \\
\text { based on } \\
\text { structured } \\
\text { approach using } \\
\text { Token Chord } \\
\text { protocol }\end{array}$ & $\begin{array}{l}\text { Based on direct } \\
\text { mobile-to-mobi } \\
\text { le technique }\end{array}$ & $\begin{array}{l}\text { Uses a DHT } \\
\text { and a } \\
\text { tree-structure } \\
\text { based on the } \\
\text { network } \\
\text { topology }\end{array}$ & $\begin{array}{l}\text { Based on } \\
\text { incentive } \\
\text { approach using } \\
\text { energy and } \\
\text { reputation of } \\
\text { peers }\end{array}$ \\
\hline $\begin{array}{l}\text { Signaling } \\
\text { Overhead }\end{array}$ & High & Medium & Low & Low & Low \\
\hline $\begin{array}{l}\text { Flexibility } \\
\text { Level }\end{array}$ & High & Low & Medium & Medium & High \\
\hline $\begin{array}{l}\text { Neighbors } \\
\text { Information }\end{array}$ & Unaware & Aware & Aware & Aware & Aware \\
\hline Drawbacks & $\begin{array}{l}\text { - Message } \\
\text { redundancy } \\
\text { - Control } \\
\text { overhead } \\
-\quad \text { Consume } \\
\text { up to } 34 \% \\
\text { from } \\
\text { bandwidth in } \\
\text { signaling }\end{array}$ & $\begin{array}{l}\text { - Not } \\
\text { Stable } \\
\text { - Possibilit } \\
\text { y of searching } \\
\text { only for exact } \\
\text { file names }\end{array}$ & $\begin{array}{l}\text { - Based on } \\
\text { social principle } \\
\text { - Applied } \\
\text { only in sharing } \\
\text { non-real-time } \\
\text { data content }\end{array}$ & $\begin{array}{l}\text { - It is not } \\
\text { stable when } \\
\text { frequent } \\
\text { disconnections } \\
\text { happen } \\
\text { - Possibilit } \\
\text { y of searching } \\
\text { only for exact } \\
\text { file names }\end{array}$ & $\begin{array}{l}\text { - Selecting } \\
\text { the best value } \\
\text { of the threshold } \\
\text { window of } \\
\text { reputation is } \\
\text { ambiguous } \\
\text { - Peers } \\
\text { share their } \\
\text { reputation } \\
\text { indexes } \\
\text { without taking } \\
\text { into account } \\
\text { security issues }\end{array}$ \\
\hline
\end{tabular}




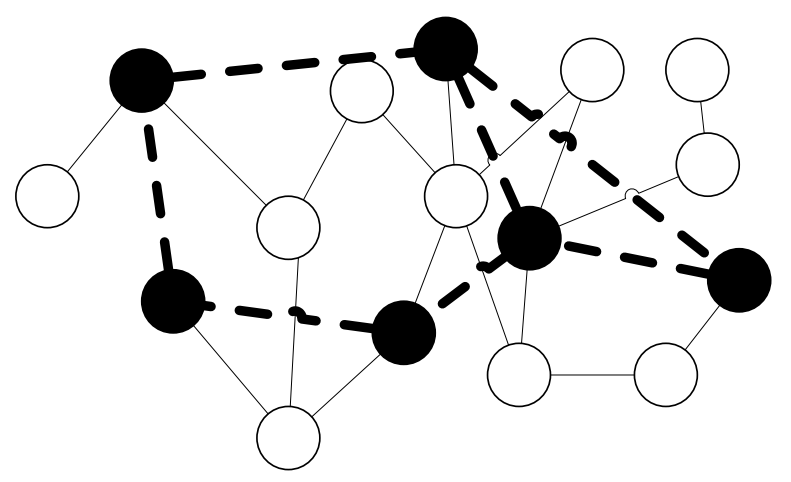

Figure 1. A P2P overlay running on a MANET

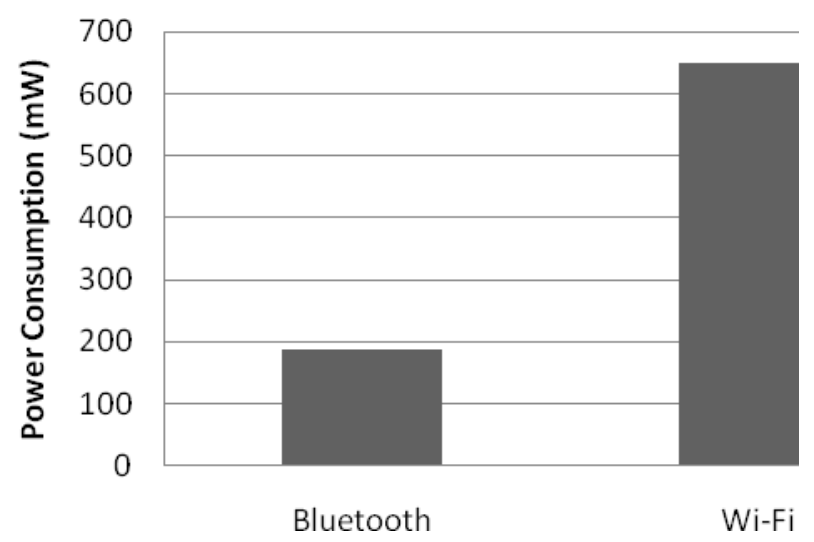

Figure 2. A power consumption comparison between Bluetooth and Wi-Fi (Palazzi et al., 2009)

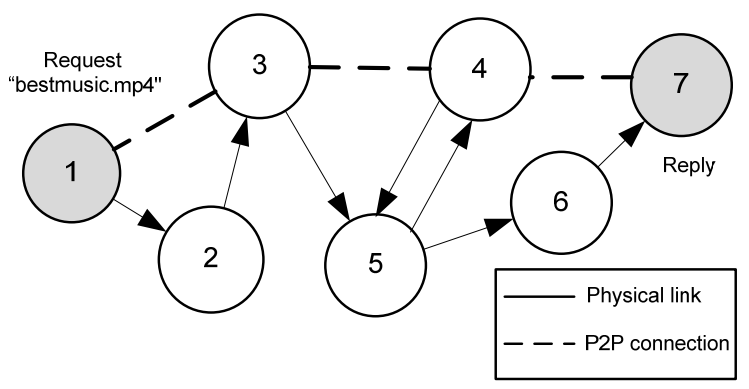

Figure 3. Gnutella file discovery process for zigzag routing problem 


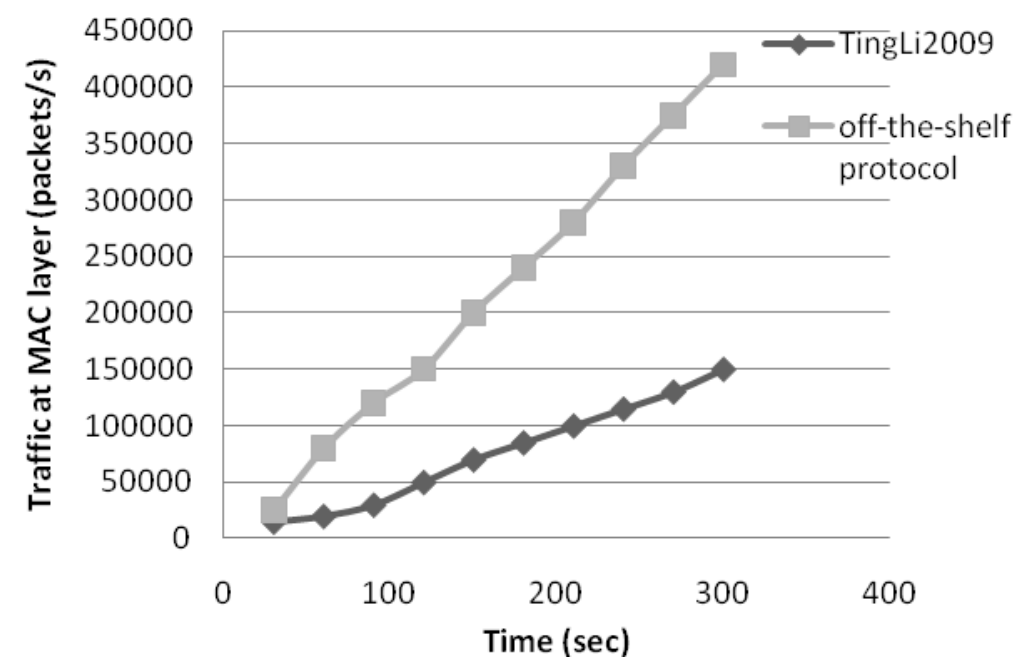

Figure 4. The traffic generated by the P2P application at the MAC layer using Ting Li2009 mechanism and off-the-shelf protocol (Ting Li et al., 2009)

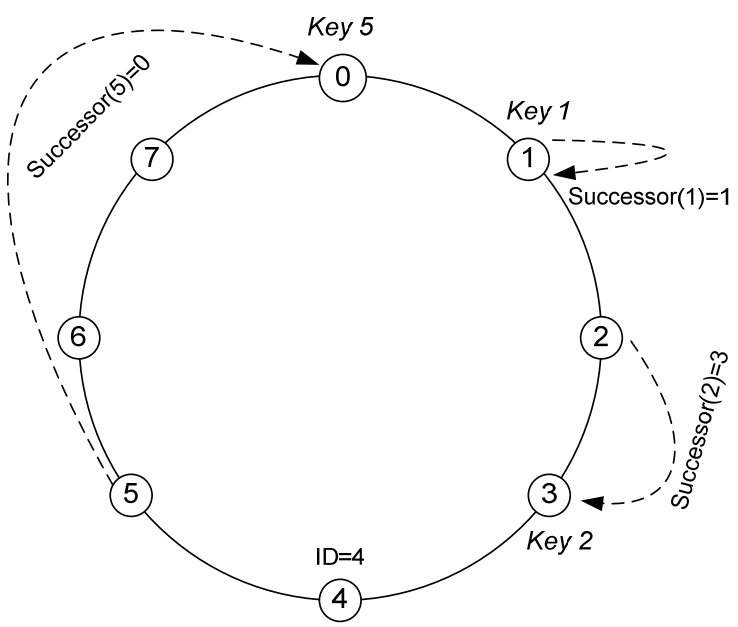

Figure 5. A Chord Identifier in a P2P network 


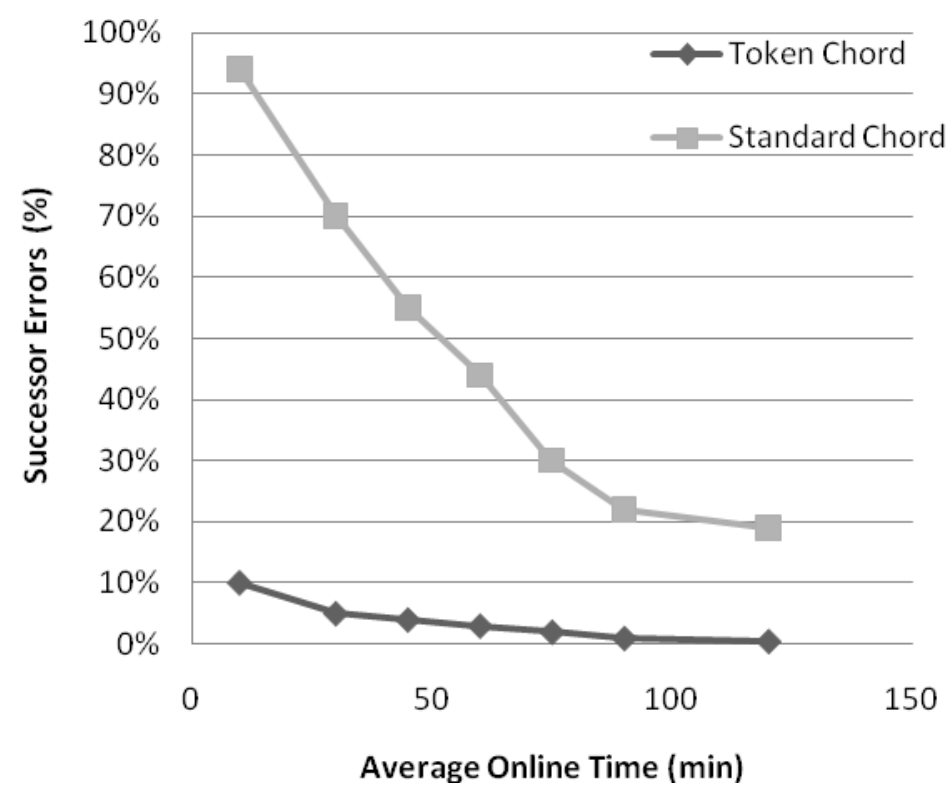

Figure 6. The performance of Token Chord compared to the Standard Chord (Kellerer et al., 2006)

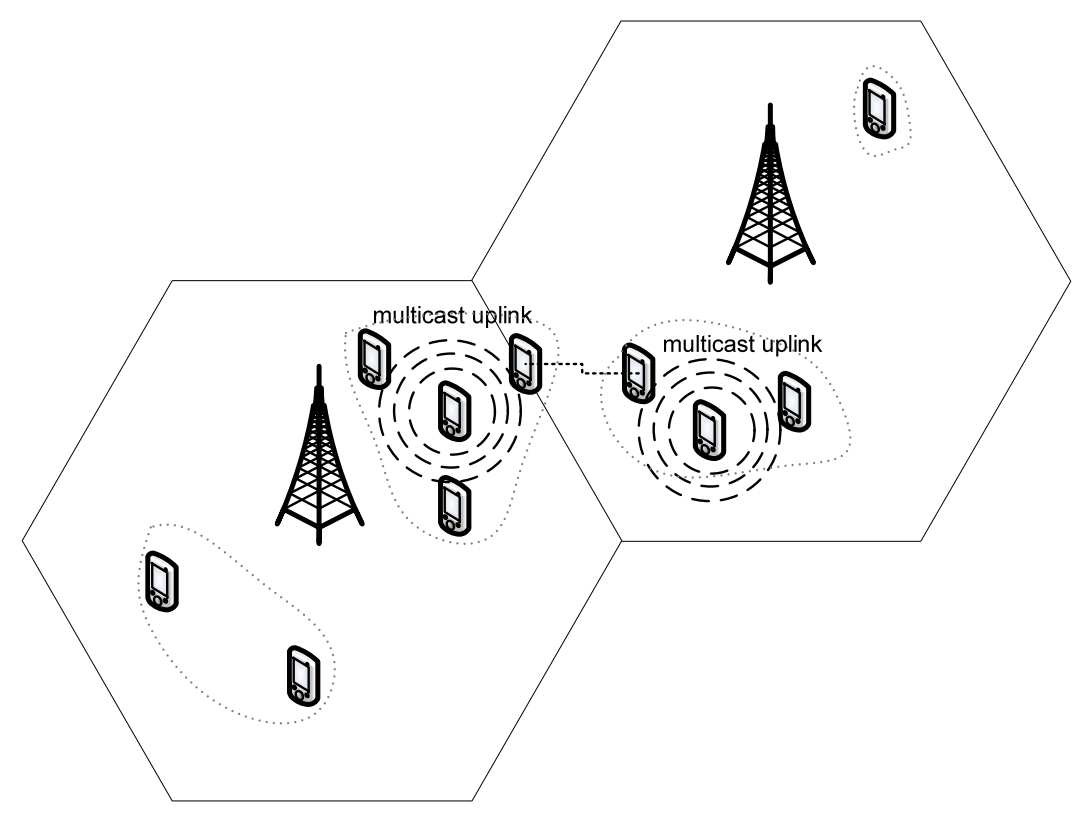

Figure 7. The concept of M2M2008 technique 


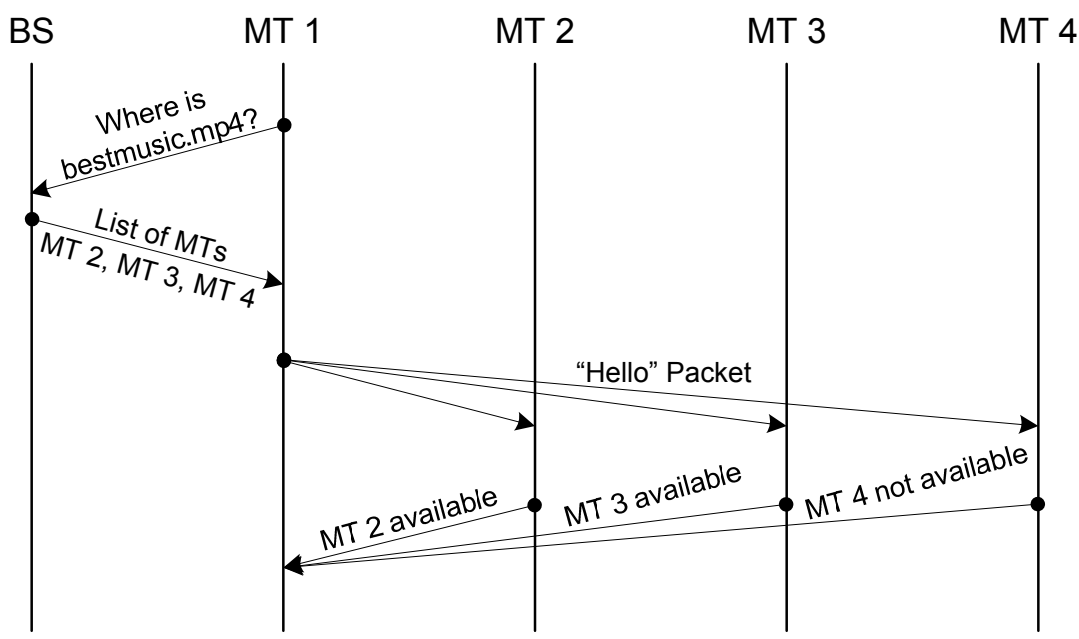

Figure 8 . The basic steps to establish a cooperative M2M2008 connection

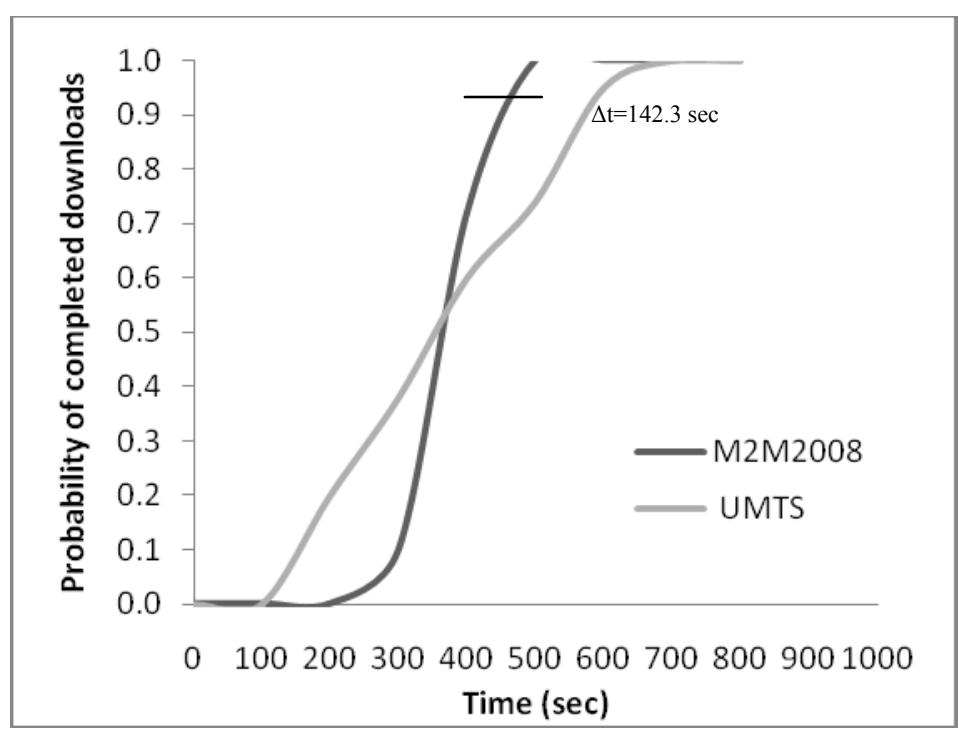

Figure 9. Comparison of download times between M2M2008 technique and conventional UMTS data transmission in high load (Popova et al., 2008) 

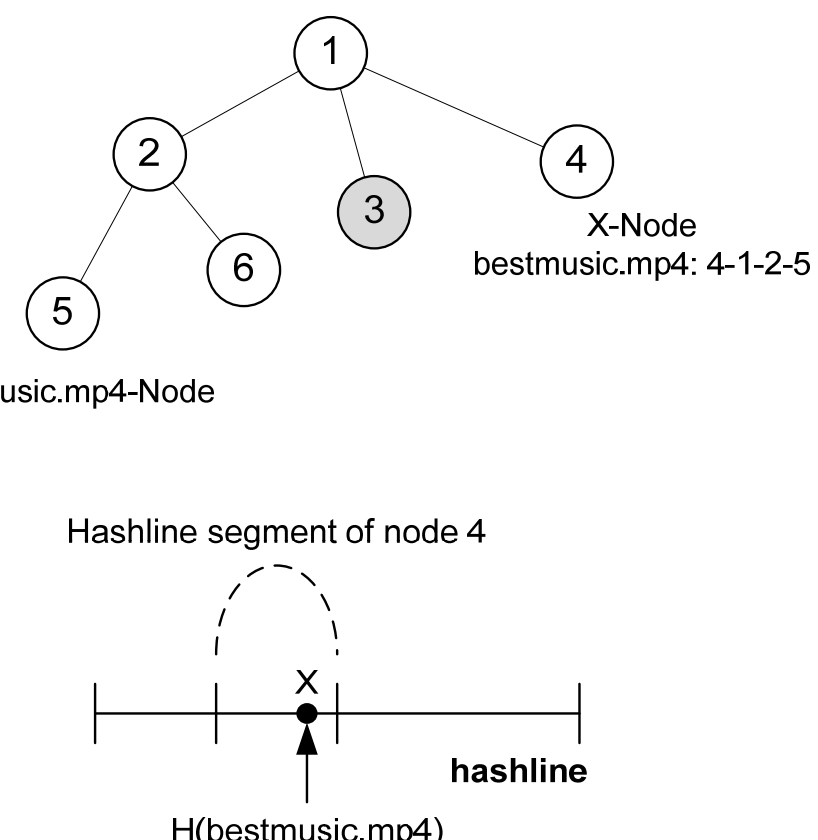

Figure 10. Using HMI2009 in MANET with six nodes 


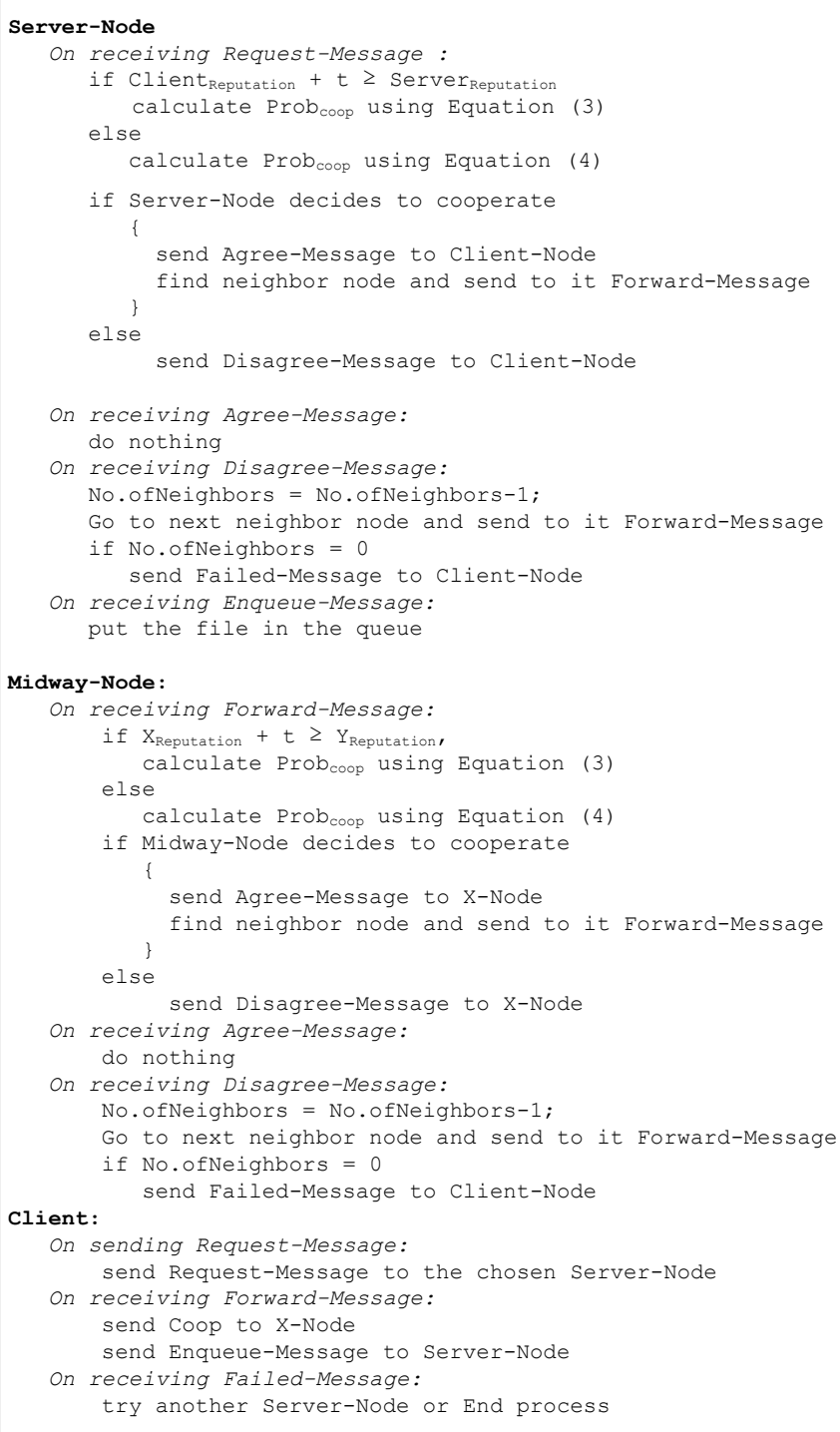

Figure 11. A pseudo-code for Incite2009 mechanism (Mawji \& Hassanein, 2009)

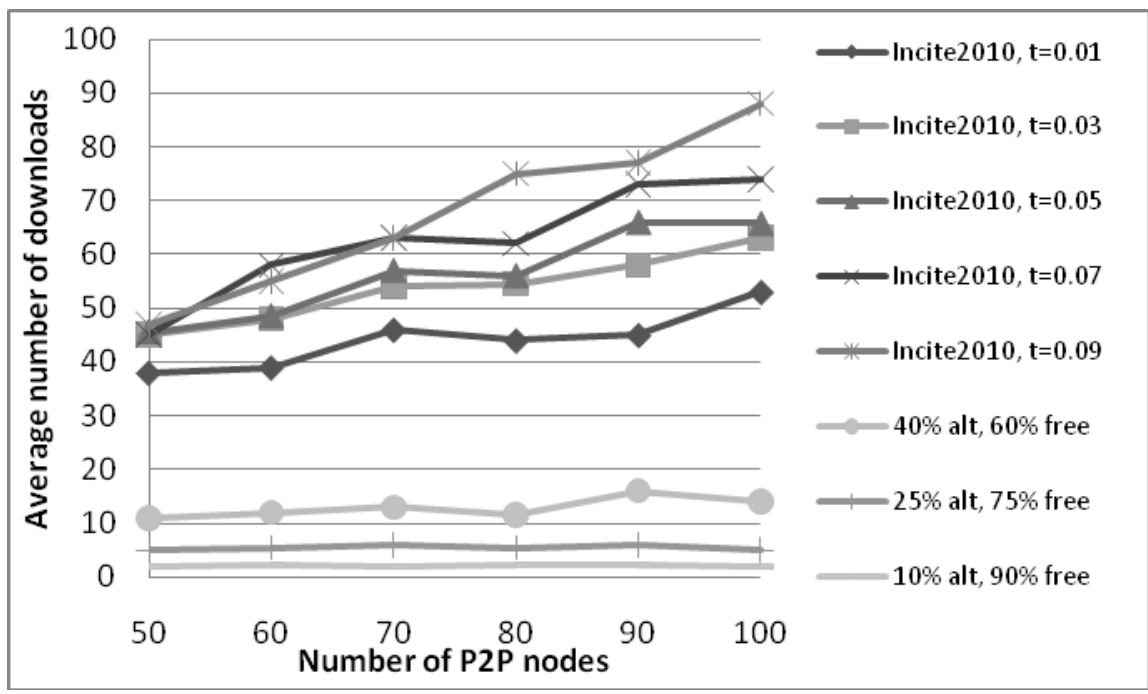

Figure 12. Average number of downloads per node (Mawji \& Hassanein, 2009) 